Disponível em

http://www.anpad.org.br/rac

RAC, Rio de Janeiro, v. 17, n. 3, art. 2,

pp. 285-303, Maio/Jun. 2013

$(\mathrm{cc})$ EY-NG

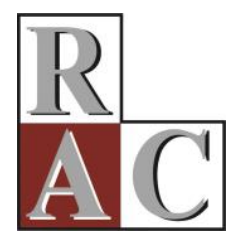

\title{
Criação de Valor: Incorporando Elementos da Economia dos Custos de Transação na Visão Porteriana
}

\section{Value Creation: Incorporating Elements from Transaction Cost Economics into the Porterian Perspective}

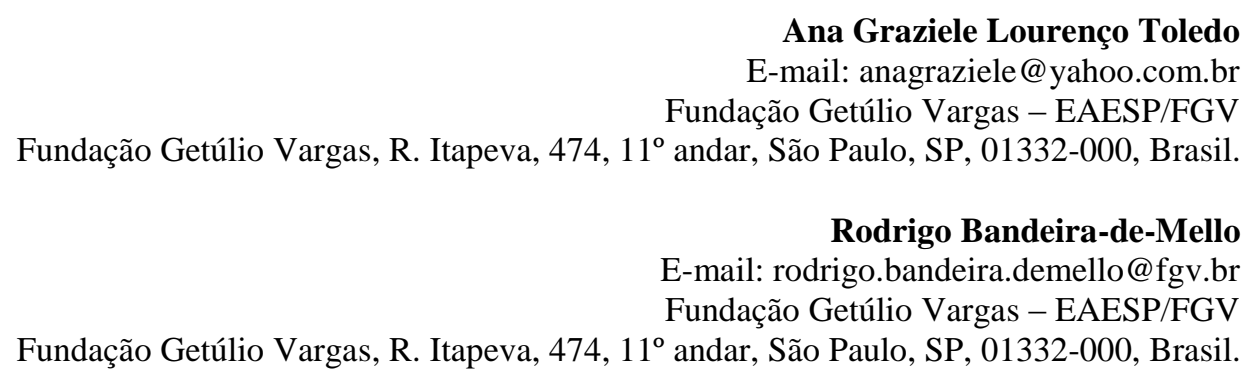

Artigo recebido em 09.04.2012. Última versão recebida em 02.08.2012. Aprovado em 24.01.2013. 


\title{
Resumo
}

Este ensaio teórico analisa o papel dos custos de transação ao longo da cadeia de valor tal como concebida na visão porteriana, objetivando propor um aparato conceitual que combine as visões da firma como um conjunto de atividades ligadas por elos e como um nexo de transações e custos que ocasionem perda de valor. Utilizandose do levantamento bibliográfico que apresenta os conceitos da vantagem competitiva de Porter e da economia dos custos de transação, elaborou-se um framework que contempla a criação de valor pela firma em três dimensões: valor criado pelas atividades, custo das atividades e custo dos elos; identificou-se também que o custo dos elos é moderado pela matriz institucional, estruturas de governança, economias de escala e escopo da firma; sendo que a incorporação destes constructos se constitui como a contribuição deste ensaio. Conclui-se que, apesar de divergentes em suas orientações para a lucratividade, a visão porteriana e a dos custos de transação apresentam potencial para integração, pois a análise da criação de valor na firma torna-se incompleta ao utilizar-se de somente uma das abordagens.

Palavras-chave: cadeia de valor; economia dos custos de transação; elos.

\begin{abstract}
This theoretical essay examines the role of transaction costs along the value chain from a Porterian perspective, aiming to propose a conceptual apparatus that combines the view of the firm as a set of activities connected by links with that of the firm as a nexus of transactions and costs that cause loss of value. Using the literature on transaction cost economics and Porter's competitive advantage, we elaborated a framework that separates value creation by a firm into three dimensions: value created by the activities, activity costs and cost of links. We also recognize that link costs are moderated by a firm's institutional matrix, governance structures, and economies of scale and scope. The incorporation of these constructs is this paper's primary contribution. We concluded that despite having differing orientations regarding profitability, the Porterian perspective and transaction cost economics have the potential for integration since analysis of value creation in the firm is incomplete when only using one of the approaches.
\end{abstract}

Key words: value chain; transaction cost economics; links. 


\section{Introdução}

A cadeia de valor é um arranjo que visa a explicar a criação e sustentabilidade da vantagem competitiva a partir da agregação de valor que ocorre nas atividades realizadas na firma, sendo a estratégia a criação de uma posição única envolvendo um conjunto diferente de atividades (Porter, 1996). A partir desta proposta e de estudos que se seguiram, entendeu-se que a agregação de valor suplantava os limites da firma e assumia características de um sistema de atividades, no qual a cadeia da firma é parte de um conjunto que envolve fornecedores, compradores e canais de distribuição ligados por elos.

As contribuições de Porter foram exploradas como se observa nos trabalhos de Shapiro, Singhal e Wagner (1993), Stabell e Fjesdstad (1998) e Patnaik e Sahoo (2009), no entanto estes estudos mantêm a concepção do valor como o produto do desempenho de um único elemento que agrega valor e desenvolve custo: a atividade. Os elos, apesar de fazerem parte da cadeia, são vistos como drivers de custo das atividades, mas não possuem, dentro da abordagem porteriana, relação com a criação de valor, demonstrando a incompletude da cadeia como aparato para análise do valor criado pela firma.

A pouca exploração do elo como variável no processo de criação de valor é uma lacuna dentro da abordagem porteriana que se procurou suprir a partir da incorporação de conceitos da economia dos custos de transação (ECT), assim, objetivando integrar as duas abordagens num framework único, utilizando-se, para tal, do levantamento bibliográfico. Tal integração, num primeiro momento, mostrase dificultosa porque se trata de abordagens que seguem orientações distintas para a lucratividade: Porter parte da estratégia para modelagem da cadeia; a ECT tem no economizing a principal ação para garantir o desempenho. No entanto, conquanto divergentes na orientação, as duas abordagens convergem ao admitirem, através de unidades de análise distintas, a existência de atividades e elos dentro da firma, e a ECT oferece elementos para explorar o papel dos elos na criação de valor.

A combinação dos conceitos de Porter com a ECT propiciou sugerir um aparato conceitual que amplia a análise proposta pela cadeia, identificando três dimensões que afetam o processo de criação de valor na firma: o valor criado pela atividade, o custo da atividade e o custo do elo, sendo a inclusão desta última dimensão a principal contribuição do artigo. Outra contribuição deste ensaio apresenta elementos moderadores na relação entre o custo do elo e a criação de valor, proporcionando maior completude à análise da firma e permitindo uma visão da criação de valor que suplanta as abordagens focadas somente no desempenho das atividades.

O trabalho foi organizado da seguinte forma: na primeira parte, serão apresentados os fundamentos teóricos sobre cadeia de valor e economia dos custos de transação. Na segunda parte, buscou-se efetuar uma integração entre as duas abordagens, elaborando hipóteses e um aparato conceitual. A terceira parte traz as implicações teóricas e gerenciais do arranjo proposto e a conclusão apresenta as limitações e oportunidades de pesquisa futura decorrentes da proposta apresentada.

Nos limites do que se propõe em um ensaio teórico, concluiu-se que a análise da criação de valor fica míope quando se utiliza apenas da lente da economia industrial ou dos custos de transação. Tal incompletude favorece distorções na análise deste processo, principalmente em relação à estrutura de custos da firma. Se uma das finalidades dos aparatos conceituais é servir de ferramenta para maximizar o desempenho, tais aparatos devem se apresentar da forma mais abrangente possível, considerando que outras dimensões também podem compor o framework analítico, como se demonstra neste trabalho. 


\section{Fundamentação Teórica}

A fundamentação teórica apresenta os conceitos que compõem as duas visões da firma que são utilizadas neste ensaio: como conjunto de atividades interdependentes e coordenadas que cria valor (Porter, 1985) e como conjunto de transações e respectivos custos, estes que diminuem o valor criado (Coase, 1937; Williamson, 1981).

\section{Cadeia de valor}

Porter (1985) assume que a firma pode ser desagregada em partes e define cadeia de valor como o conjunto sequenciado de atividades diferenciadas e interdependentes de uma firma que compõem a produção de um bem ou prestação de um serviço visando à criação de valor. Shapiro et al. (1993) consideram a cadeia de valor como um processo logístico que coordena as atividades em nível detalhado e operacional através de fluxos de valor e informações que correm entre fornecedores e clientes, intermediados por processos internos e externos, transcendendo o nível da firma e agregando outros participantes da indústria. Tal coordenação é vista como eficácia operacional que não propicia vantagem competitiva, mas participa de sua criação (Porter, 1996).

A composição da cadeia abrange todas as atividades desenvolvidas na firma, diferenciando-se de acordo com o valor que agregam em termos de posição de custo ou diferenciação. Assim, existem as atividades primárias que compõem a criação física, comercialização, entrega, suporte e assistência do produto; e atividades de apoio que proporcionam suprimento de insumos; e infraestrutura que sustentam todas as atividades da cadeia (Porter, 1985). Considerando a interdependência entre as atividades, existe uma segunda classificação: atividades genéricas - o valor é independente do contexto, possuindo uma configuração ótima para todas as firmas; ou atividades especificamente estratégicas, quando o valor é afetado pelas outras atividades, sendo necessária uma configuração ótima específica para cada firma (Porter \& Siggelkow, 2008).

Mesmo reconhecendo a existência de um processo de coordenação, as definições apresentadas situam nas atividades o locus da criação de valor e vantagem competitiva, não mencionando as perdas de valor que ocorrem ao longo da cadeia. Tal constatação torna-se mais evidente no sistema de atividades que inclui, além da firma produtora, os outros atores econômicos presentes na indústria.

\section{Sistema de valor}

A Figura 1 mostra a configuração genérica de um sistema de atividades composto pelo produtor e suas interfaces a montante (fornecedor) e a jusante (distribuidor), onde as cadeias de valor são ligadas por elos que são os pontos de contato entre as unidades do sistema (Stabell \& Fjestad, 1998).

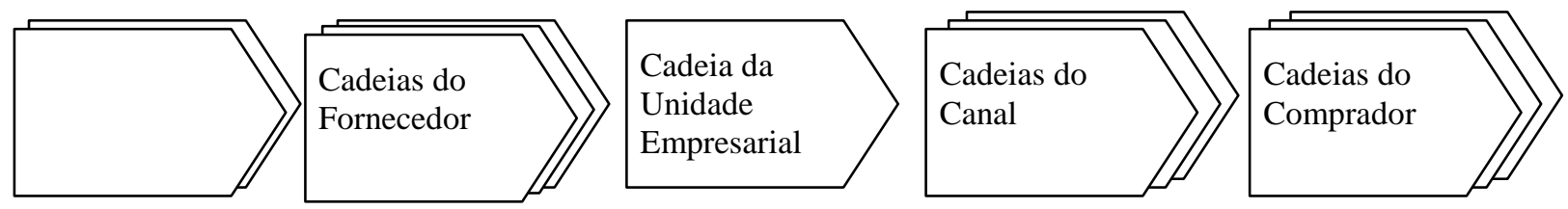

Figura 1. Sistema de Valor.

Fonte: adaptado de Porter, M. E. (1989). Vantagem competitiva (p. 32). Rio de Janeiro: Campus.

Assumindo que a vantagem competitiva advenha do posicionamento e guarde relação com o escopo (Porter, 1989), a modelagem do sistema de atividades deve considerar:

o posicionamento adotado pela firma uma vez que: (a) cada atividade tem sua própria estrutura de custo e pode ser reestruturada para minimizar os custos em comparação com a concorrência; (b) todas as atividades oferecem potencial de diferenciação através de condutores como políticas e integração (Porter, 1989); 
dados e informações gerados por todas as atividades e que dentro delas circulam (Shapiro, Shingal, \& Wagner, 1993);

fatores estruturais que determinam o comportamento de custo das atividades de valor, os drivers de custo, sendo o maior deles a escala; fatores externos, evidenciando-se a influência da tecnologia e assumindo que as atividades representam um terço da totalidade, tendo no valor da venda e no valor da rede os complementos para criação de valor em nível da firma (Stabell \& Fjesdstad, 1998);

a estrutura setorial e a complexidade do produto, bem como o impacto no sistema, sendo que atividades contextualizadas também podem conduzir a diferentes posicionamentos estratégicos (Porter \& Siggelkow, 2008); e,

o grupo estratégico (Patnaik \& Sahoo, 2009).

Considerando a existência dos condutores e da contextualidade, deduz-se que, além das atividades, a modelagem de um sistema deve conter elementos que conectem estas atividades, dessa maneira, proporcionando sinergia ao conjunto: o elo. Observa-se, portanto, que há o reconhecimento de que as unidades básicas da constituição de uma cadeia/sistema são as atividades e os elos, mas mantém-se a ideia de que a criação de valor reside nas atividades e no seu desempenho, permanecendo o elo, uma lacuna pouco explorada no aparato conceitual porteriano.

\section{Elos}

Até o momento, a visão porteriana reconhece a existência dos elos e seu papel como conexões existentes ao longo de toda cadeia/sistema por onde ocorrem as passagens dos outputs de cada atividade. A principal causa para o surgimento dos elos seria a necessidade de integração entre as atividades organizacionais, proporcionando maior eficácia operacional a partir do estabelecimento de uma relação de complementaridade entre as cadeias de valor envolvidas, desta forma, admite-se que o elo pode ser fonte de vantagem competitiva (Porter, 1989).

Porter (1989) acrescenta que o elo não é a integração entre as atividades, mas o modo como esta integração dá-se, que ocorre pelo estabelecimento de uma estrutura organizacional que afeta a estrutura de custos ou se torna condutora de diferenciação. Esta estrutura pode ser afetada pela intensa integração das atividades que impossibilita identificar onde termina uma e começa outra, pela negligência de ações importantes ou pela existência de barreiras organizacionais (Porter, 1989), como a definição do escopo da firma (Stabell \& Fjesdstad, 1998).

Posteriormente, a visão porteriana parece retroceder em relação ao papel do elo como fonte de vantagem competitiva, voltando à crença inicial de que o valor é criado pelas atividades. Porter e Siggelkow (2008) afirmam que a consistência interna propiciada pelos elos é necessária, mas que somente contribui para a criação de valor e proteção da vantagem competitiva, que são, ambas, realizadas pelo desempenho das atividades modeladas a partir da exploração de trade-offs da indústria.

\section{Lacunas existentes nas concepções de cadeia de valor/sistema de atividades}

Quando Porter (1989) admite que o valor total criado pela firma é a diferença entre o valor criado e o custo das atividades, conclui que, mantendo-se o desempenho das atividades, a minimização dos seus custos favorece o aumento do valor total. Ocorre que, mesmo admitindo a existência dos elos e reconhecendo a necessidade de consistência interna, os aparatos porterianos - cadeia de valor e sistema de atividades - permanecem considerando somente os custos das atividades e ignorando os custos dos elos; não se sabe se os custos dos elos estão embutidos nas atividades, se esses custos afetam o valor total criado ou a influência dos elos sobre o desempenho da cadeia/sistema. A falta de exploração dessas lacunas tornou a cadeia/sistema um aparato útil, porém incompleto para análise da criação de valor. 
As contribuições da cadeia/sistema citadas na literatura seguem a concepção porteriana de criação de valor a partir das atividades: obtenção da vantagem competitiva por meio da criação e sustentabilidade da cadeia, do auxílio ao projeto de estrutura organizacional e departamentalização sistematizada e da compreensão da origem do lucro da firma (Porter, 1989); entendimento e estabelecimento de vantagem competitiva baseada em custos, na determinação de estratégias de coordenação de diferentes atividades e na assistência na análise dos custos e diferenciação (Shapiro et al., 1993); auxílio na análise no nível da firma acerca de forças e fraquezas (Stabell \& Fjesdstad, 1998); e identificação de atividades que criam e que não criam valor, propiciando uma liberação de recursos para ser melhor utilizados (Patnaik \& Sahoo, 2009). Ressalta-se que não há nestas observações menções feitas aos elos.

Mesmo não explorando o papel dos elos na firma, Porter oferece um indicativo do seu comportamento: reconhece-os como drivers de custo, mas não como unidades criadoras de valor. Somando-se isso à definição que traz o elo como ponto de conexão onde ocorre passagem de outputs, sugere-se que a exploração do papel dos elos no processo de criação de valor da firma possa se dar utilizando o aparato teórico da economia dos custos de transação, que compartilha do entendimento que tais conexões não criam valor, mas contribuem para a criação se forem desempenhadas de forma econômica.

\section{Custos de transação}

Ronald Coase (1937) deu início ao estudo da economia dos custos de transação (ECT), então, admitindo a existência de custos além dos tradicionalmente tratados pela economia industrial. Reflexões foram aprofundadas em trabalhos de Williamson (1981, 1991a, 1991b) e, apesar de criticada por não considerar aspectos da organização (Lindenberg, 2003) como estruturas informais, regras culturais, normas sociais e redes (Voss, 2003), a ECT aborda um nível de análise semimicroanalítico para compreender o impacto das transações nas estruturas de custo das firmas.

\section{Transação e custo de transação}

Transações são compostas por operações e desenvolvem custos para serem realizadas; definemse como custos de transação aqueles próprios do desenvolvimento da transação, ou seja, aquilo que se gasta ao efetivar a transferência de um bem (Coase, 1937). Inerente a esta definição, nenhuma transferência de bem se dá sem custo, visto que são verificados custos na busca, informação, negociação, decisão e execução de uma operação, ou seja, nenhuma transação é livre de ônus, que, em excesso, é resultado de organização inferior e operações mal-adaptadas (Coase, 1937).

Para Coase (1937), os custos de transação é que definem a firma. O mercado, entendido como provedor eficiente pela teoria econômica clássica, disponibiliza ativos para serem consumidos a um determinado custo. Enquanto o custo da aquisição dos ativos do mercado é alto, a firma surge como um conjunto de transações de longo prazo que são menos custosas que aquelas a curto prazo, representadas pelas relações de mercado, ficando estas sujeitas a gerar maiores custos de transação. Observa-se aqui que não só a transação em si, mas seu tempo de duração determina os custos que tendem a ser minimizados pela firma, uma vez que são diluídos em um tempo maior. A ocorrência de uma transação se dá em face da existência de partes, as quais Williamson definiu como atividades: entende-se que uma transação ocorre quando um bem ou serviço é transferido de uma atividade para outra, sendo estas atividades delimitadas por uma interface tecnológica separada (Williamson, 1981) a cada nível organizacional.

Segundo Williamson (1981), a análise dos custos de transação desenvolve-se em três níveis: a estrutura do negócio, as partes operacionais - quais atividades devem ser desempenhadas pela firma e com qual finalidade - e a organização dos ativos humanos. Cada nível utiliza-se de formas de controle para a realização de suas transações, que variam de acordo com a natureza tecnológica das atividades. Nesta visão, a análise dos custos de transação se constitui como o elemento mais importante na 
estratégia de negócios, pois a estratégia é dependente dos custos que representam a eficiência, ou seja, o foco econômico na visão da ECT (Williamson, 1981, 1991a, 1991b).

\section{Recursos da economia dos custos de transação}

Williamson (1981) acredita que a alta performance econômica é dada em face da incerteza e da capacidade para adaptar as atividades de forma eficiente, conduzindo à adaptação das estruturas que as governam. No entanto, firmas estão sujeitas à incerteza, o que faz o instrumento de governança utilizado para o controle das atividades ser mais complexo e custoso para ser determinado e cumprido; este instrumento que media as trocas econômicas é o contrato (Williamson, 1981), seja ele formal ou informal, cuja elaboração seria pautada pelas seguintes dimensões:

1. crenças de comportamento, entendidas como oportunismo e racionalidade limitada que geram, respectivamente, a necessidade por contratos e sua incompletude; sujeitos a estas crenças, os contratos podem contar com a adoção de salvaguardas para combater limitações de formulação/resolução, preferências gerenciais e ações oportunistas dos concorrentes (Williamson, 1985);

2. dimensões ou atributos das transações, entendidas como incerteza, frequência de ocorrência e especificidade de ativos. Destas dimensões, destaca-se o papel da especificidade de ativos (Williamson, 1985), que deve ser considerada no processo decisório sobre a forma organizacional a ser assumida pela firma, uma vez que pode incorrer em altos custos para seu estabelecimento; e

3. recursos-chave de governança, que tratam do conhecimento das microforças próprias dos custos de transação e que determinam formas reduzidas de estresse das variáveis importantes, identificando atributos de desempenho, assumindo que os agentes que transacionam entre as atividades possuem limitações cognitivas e que, portanto, atores humanos são um atributo-chave para a determinação das estruturas de governança (Williamson, 1999).

\section{Especificidade de ativos}

Alchian e Demsetz (1972) veem as firmas como espécies de mercados altamente especializados e capazes de desempenho econômico superior à utilização do mercado externo devido a um hipotético custo mais baixo do conjunto de insumos específicos que processam. Esse conjunto de insumos fornece, a partir de vários tipos de recursos, respostas específicas de produtividade, tornando o produto não a soma de resultados de cada recurso, mas o desempenho do conjunto de ativos especializados. Ativos possuem aplicações definidas, porém, também, podem ser utilizados em uma situação e desenvolver melhor desempenho nela do que em outra, contribuindo para geração de quase-renda (Klein, Crawford, \& Alchian, 1978). Contudo a realocação de um ativo incorre em custos de transação pela necessidade de refazer a estrutura de governança utilizada para controlá-lo e, desta forma, a escolha ótima da especificidade de ativos requer uma análise simultânea entre custos e demanda pelo ativo, determinando a natureza das relações contratuais da firma, levando-a, muitas vezes, à introdução de especificidade em ativos requeridos em grande quantidade (Williamson, 1981).

Williamson (1981, 1991a, 1991b) sugere diferentes comportamentos dos agentes diante da especificidade dos ativos, comportamentos estes que representam as conexões entre firma e mercado: os poucos específicos, geralmente, são contratados do mercado; os relativamente específicos seriam desenvolvidos em mercados bilaterais ou híbridos como alianças estratégicas; os mais específicos seriam produzidos pela firma - as hierarquias. Esta decisão é mediada pelos custos de transação envolvidos na especificidade dos ativos e no entendimento de quanto valor se perde quando se utiliza um ativo em uma situação para a qual ele não é específico e o que isso afeta na geração de quaserenda.

Ativos pouco especializados provocam um comportamento oportunista nos envolvidos na transação que, utilizando do poder de mercado, buscam eficiência através das oportunidades que lhes 
são oferecidas; neste caso, a pouca especificidade de um ativo reduz a dependência do mesmo por outras firmas e, consequentemente, seu valor de capital. Já a alta especificidade aumenta o risco envolvido nas transações e os esforços de compradores e fornecedores para construir transações cuja continuidade e durabilidade sejam maiores, o que colabora positivamente para a sustentabilidade de uma eventual vantagem obtida por este atributo (Williamson, 1991a). A especificidade dos ativos é, então, definida pela perda de valor produtivo que ocorre quando um ativo tem seu uso redirecionado para outro fim alternativo e pode surgir da localização, das estruturas de governança, da condição física, do componente humano e da demanda do ativo, gerando uma dependência bilateral (Williamson, 1996).

Apesar da maior especificidade, minimizar perdas associadas a ações oportunistas também dificulta processos de adaptação que a firma venha a desenvolver na busca pela manutenção de sua competitividade, uma vez que mudanças poderiam envolver a realocação desses ativos que quanto mais especializada mais custosa tornará a modificação na sua forma de governança. Assim, a especialização dos ativos só deve ser realizada quando realmente contribuir com a redução dos custos de produção ou adição de renda (Williamson, 1996).

\section{Lacunas existentes nas concepções da economia dos custos de transação}

A ECT oferece um aparato conceitual que permite explorar a participação dos custos na estrutura de criação de valor na firma a partir da identificação das transações e dos custos que são inerentes a elas e que nascem da necessidade do estabelecimento de estruturas de governança, salvaguardas e especificidade de ativos. Reconhece-se que a transação ocorre nos níveis de negócios, operacional e ativos humanos (Williamson, 1981), mas especificamente no nível operacional, a ECT procura determinar quais atividades comporão a hierarquia a partir do custo das transações que estas atividades demandam, não considerando o valor que elas agregam com seu desempenho. Esta lacuna pode ser preenchida utilizando o aparato da cadeia de valor que enfatiza o papel das atividades.

Tal orientação surge porque a ECT vê a firma como uma forma organizacional mais econômica em relação ao mercado e não como uma função criadora de valor, pois seu foco está na transação e não na atividade. Nesta perspectiva, entende-se que a transação só cria custos e que seu valor é determinado por ser mais ou menos econômica; o valor, então, assume-se como resultado da eficiência tecnológica obtida pela estrutura de governança, ou seja, o valor é criado através da redução dos custos e não da agregação de valor feita nas operações que compõem as transações.

Ao contrário de Porter, a ECT adota o economizing como perspectiva para criação de valor; o strategizing surge em decorrência da opção pelo menor custo, ou seja, as atividades são conformadas após a decisão sobre a forma organizacional mais econômica. Este posicionamento revela a dificuldade na integração entre ECT e a abordagem porteriana, mas, ao mesmo tempo, sugere que um aparato que considere elementos de ambas as visões pode contribuir para uma análise mais abrangente do processo de criação de valor na firma.

\section{O Aparato Proposto: Custos de Transação dos Elos}

O item anterior apresentou os conceitos de cadeia de valor/sistema de atividades e custos de transação que são usados neste ensaio de forma instrumental. Sugere-se aqui que strategizing e economizing devem ser condição necessária para criação de valor, ambos expressos pela capacidade de adaptação da firma (Williamson, 1981), configuração organizacional em termo de estruturas alternativas de governança (Williamson 1985; 1991b) e exercício das opções excludentes (Porter, 1996).

As abordagens teóricas citadas usam níveis e unidades de análise diferentes, mas também admitem que firmas ou sistemas possuem algum elemento que intermedeia as diferentes atividades de 
criação de valor: esse elemento é o elo. Conforme representado na Figura 2, em um sistema genérico com $n$ atividades, uma dada atividade $A_{i}$ agrega valor $\mathrm{V}_{i}$, mas também incorpora um custo $\mathrm{C}_{\mathrm{i}}$. $\mathrm{A}$ diferença entre $V_{i}$ e $C_{i}$ resulta na margem $M_{i}$ criada pela respectiva atividade. $O$ somatório das margens de todas as $n$ atividades resulta no valor total (VT) criado pelo sistema de atividades (Equação 1). A atividade $A_{i}$ e sua atividade sucessora $A_{i+1}$ são ligadas por um elo $E_{i, i+1}$. A quantidade de elos em um sistema sempre será igual a $n-1$ atividades. Para a economia industrial, os elos são pontos de contato entre as atividades e parte da interface tecnológica que as une, sendo compostos por operações que manipulam ativos de forma coordenada; nos custos de transação, os elos são transações comandadas por estruturas de governança e responsáveis pelos resultados diferenciados das firmas. As estruturas de governança determinam o dimensionamento da cadeia de valor à medida que são capazes ou não de gerenciar as transações, podendo constituir-se como custos ex ante que visam a prevenir custos ex post (Williamson, 1985) e que atuam como forma de adaptação da firma, que é o problema central da organização econômica (Williamson, 2008).

Mesmo diante desta constatação, na economia industrial, assume-se que a vantagem competitiva é obtida pela firma através da criação e entrega de valor, que é "o montante que os compradores estão dispostos a pagar por aquilo que a empresa oferece" (Porter, 1989, p. 34), medido pelo preço que o comprador se dispõe a pagar dada sua compreensão sobre os atributos do produto e seu papel na minimização de custos ou otimização de desempenho (Porter, 1989; 1990), diferenciando-se da abordagem do valor adicionado, em que o objetivo é maximizar diferença entre compras e vendas (Shank \& Govindarajan, 1992).

Considerando que a estrutura da indústria implica as mesmas influências para todos os concorrentes, a criação de valor na visão da organização industrial está relacionada com a configuração da cadeia coerente com a posição competitiva escolhida, ou seja, modelar atividades de forma que seu desempenho resulte em maior valor criado do que custo demandado. Portanto, o locus da criação de valor é na atividade, conforme a Figura 2.

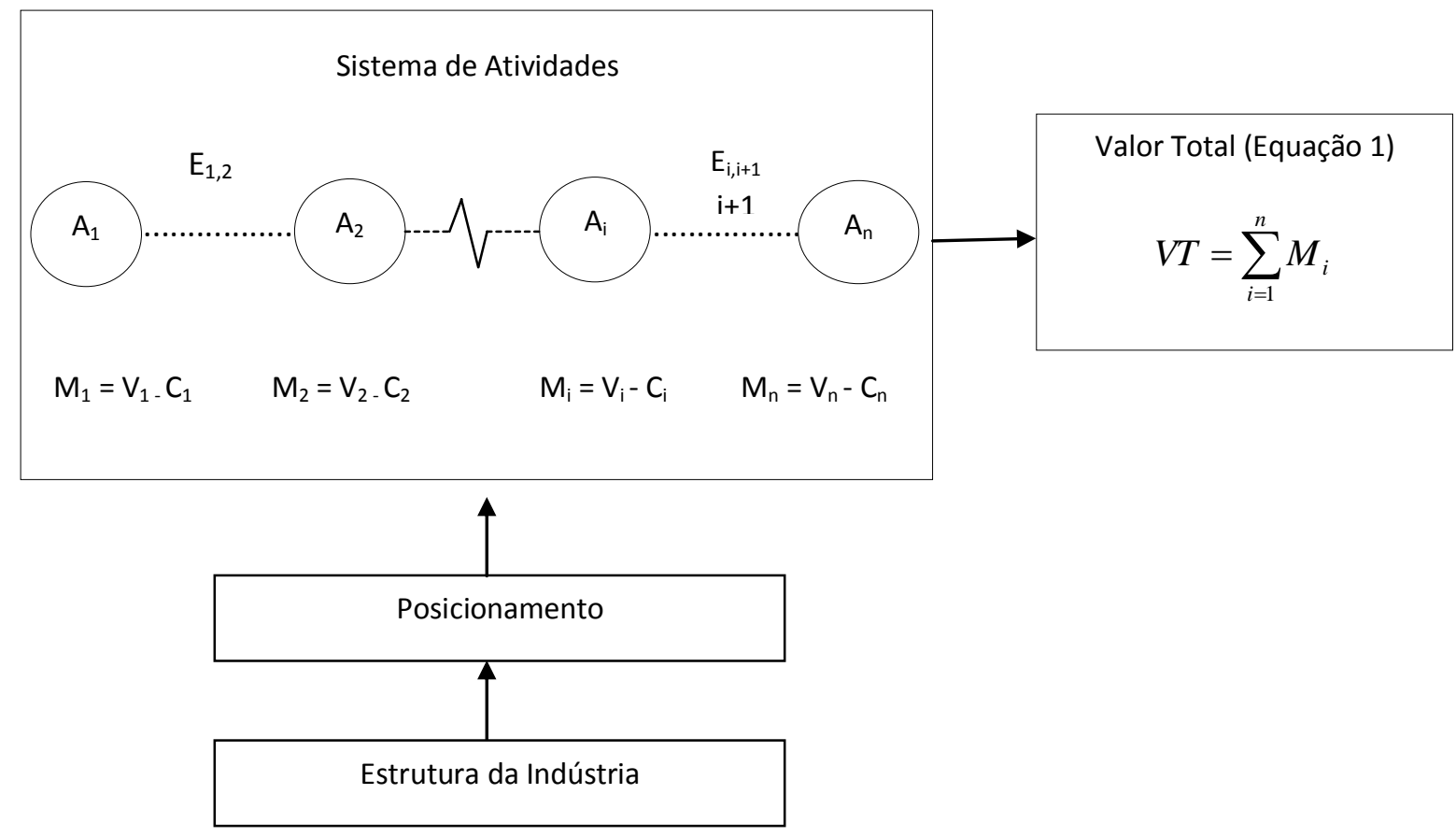

Figura 2. Criação de Valor Segundo a Cadeia de Valor Tradicional.

Fonte: Elaborado pelos autores.

Organizações que pertençam a mesma indústria competem pelo mesmo mercado e tenham os mesmos fornecedores podem modelar cadeias diferentes (Shank \& Govindarajan, 1992), pois tem-se que a vantagem competitiva só é possível através do estabelecimento de uma diferença preservável entre a firma e seus concorrentes em termos de fornecimento de valor, residindo na escolha deliberada 
de uma cadeia única a partir da opção excludente (Porter, 1996). Aliada à perspectiva dos custos de transação, a unicidade da cadeia se verifica em quão específicos são os ativos envolvidos, propiciando uma cadeia diferente dos rivais, alimentando a crença de que a vantagem competitiva acontece em determinadas realidades, constituindo-se como casos únicos (Powell, 2001). Desta forma, a exploração de trade-offs é uma condição necessária, porém não suficiente para a criação de valor na cadeia e deve ser acompanhada pelo custo dos elos.

A cadeia de valor/sistema de atividades reconhece a existência do elo como uma de suas unidades e o considera como um dos drivers de custo das atividades (Shank \& Govindarajan, 1992); entre estas atividades, ocorrem transações cuja qualidade é medida pelo custo e desempenho. Desta forma, quanto mais econômica forem as transações, maior valor será criado na cadeia/sistema, garantindo a manutenção da vantagem competitiva através de lucros de curto e longo prazo (Shapiro et al., 1993). O gap no aparato porteriano ocorre quando os custos das transações entre as atividades não são considerados, o que modifica a estrutura da criação de valor, considerando que nenhuma transação é isenta de custos, uma vez que atividades possuem superfícies de contato imperfeitas que criam friç̧ões, ou seja, links incompletos da cadeia de valor da indústria que mantêm algumas partes em contato, limitando a habilidade de encontrar alternativas estratégicas (Chatain \& Zemsky, 2011). Então, deve participar da criação do valor total na cadeia, além dos elementos sugeridos no framework original, o custo dos elos conforme a equação 2 , de onde se pode concluir que, mantendo-se o desempenho das atividades, quanto maior forem os custos dos elos menor será o valor total criado. Com base nisto, apresenta-se a hipótese (H1), conforme Figura 3:

H1: o valor total (VT) de uma cadeia é negativamente relacionado com o custo dos elos $\left(\mathrm{CT}_{\mathrm{i}, \mathrm{i}+1}\right)$.

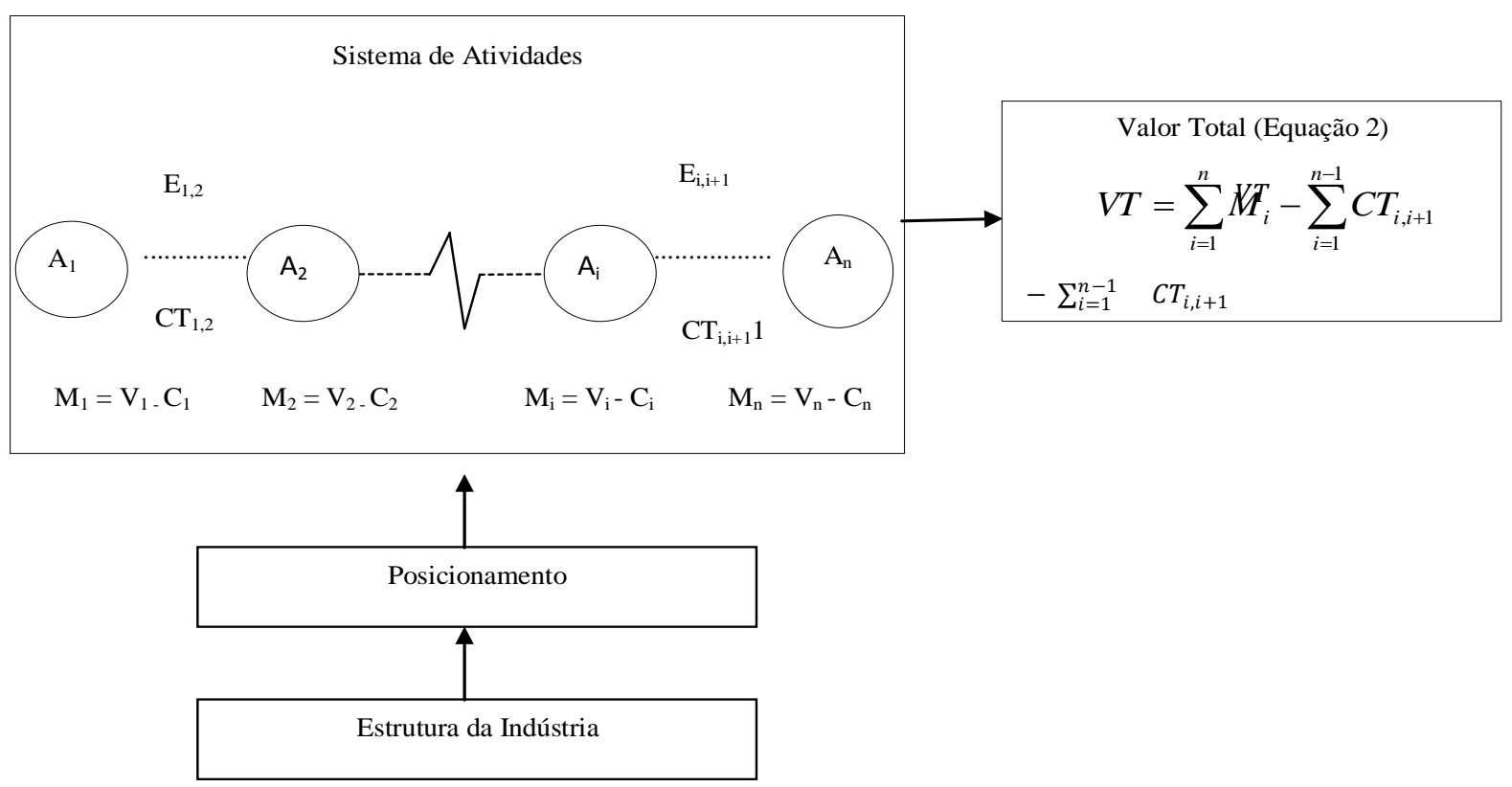

Figura 3. Integrando a Abordagem Porteriana e a ECT. Fonte: Elaborado pelos autores.

Uma competição que possua bases mais específicas e difíceis de serem emuladas pode garantir maior valor para a firma, sendo que a eficiência não ocorre pelo melhor recurso, mas pelo desempenho produtivo relativo do conjunto de recursos (Alchian \& Demsetz, 1972), o que se dá pelos elos cuja administração é uma tarefa organizacional mais complexa do que a administração das atividades, afirma Porter (1989). Essa constatação justifica-se pelo fato de que quanto mais específico for o elo, maior a dificuldade em copiá-lo e geri-lo de forma a propiciar benefícios como níveis adequados de estoques, tempos de espera, diminuição do comportamento oportunista, facilitação da comunicação, prestação de serviços auxiliares ou de apoio. 
Porter (1989) admite que a sustentabilidade pode ser garantida pelo pioneirismo e pelo número de possibilidades de reconfiguração da cadeia de valor, tornando-a diferente, específica e de tamanho adequado, o que leva a crer que, apesar de única e específica, esta cadeia deve ser flexível para se adaptar. No entanto há dois entendimentos sobre o papel das atividades: (a) o nível de agregação de valor se dá pela especificidade das atividades enquanto tecnologia e estratégia distintas (Porter, 1989); (b) são as unidades que concentram a criação de valor (Porter, 1996). Ocorre que a competição na base das atividades fica restrita à eficácia operacional que expande o valor criado para todos os participantes da indústria, além de ser alvo para benchmarking, o que iguala as empresas entre si, tornando as atividades mais genéricas (Porter, 1999). Aqui os elos surgem como complemento à eficácia operacional das atividades sendo que existem, respectivamente, elos mais adequados para cada tipo de situação (Roodhoof \& Warlof, 1999) e mais eficientes (Porter, 1999) para que, na integração vertical, os custos para adequar as fricções através da adoção de padrões sejam menores, estabelecimento da reputação e uso da tecnologia (Chatain \& Zemsky, 2011).

Nesta visão, a coordenação de atividades e elos deve ser feita por uma forma de governança que surge após a definição da matriz institucional a ser adotada na cadeia de valor (Gereffi, Humpfrei, \& Sturgeon, 2005) cujos custos, segundo a ECT, variam de acordo com os atributos das transações; isso reforça o argumento porteriano de que devem existir configurações diferentes e mais adequadas para cada firma, considerando que tais formas podem desenvolver sunk costs e reduzir a capacidade, como é o caso das hierarquias (Williamson, 2008). Operacionalizando a definição de Williamson, ao se optar por uma forma de governança deve-se considerar o custo de cada uma destas formas que é, conforme equação 3 , a soma do custo das atividades $\left(\mathrm{CA}_{\mathrm{i}}\right)$, das crenças de comportamento e dos atributos das transações que são representados pelo oportunismo $\left(\mathrm{COP}_{\mathrm{i}, \mathrm{i}+1}\right)$, racionalidade limitada $\left(\mathrm{CRL}_{\mathrm{i}, \mathrm{i}+1}\right)$ e pela especificidade de ativos $\left(\mathrm{CEA}_{\mathrm{i}, \mathrm{i}+1}\right)$; tornando o custo de uma matriz institucional $\left(\mathrm{C}_{\mathrm{MI}}\right)$ a soma destes de forma que

$$
C M_{i}=C A_{i}+C O P_{i, i+1}+C R L_{i, i+1}+C E A_{i, i+1} \quad(\text { Equação } 3)
$$

A especificidade dos ativos é um dos atributos das transações e, conforme indica a ECT, a presença de alta especificidade atrai o comportamento oportunista, propiciando maiores custos nas transações. A especificidade dos ativos é um dos atributos das transações e, conforme indica a ECT, a presença de alta especificidade atrai o comportamento oportunista, propiciando maiores custos nas transações. Especificidade de ativos e crença no comportamento assumem valor maior que zero, sendo o que: (a) quanto maior o $\mathrm{C}_{\mathrm{MI}}$, indica-se a adoção do mercado, e (b), quanto menor o $\mathrm{C}_{\mathrm{MI}}$, indica-se a adoção da hierarquia (Williamson, 1985). Apesar das atividades serem constituídas de forma única na cadeia, o que incorre em alta especificidade de ativos e conduz à hierarquia, Porter admite a utilização do mercado ou híbridos porque considera que um maior número de atividades implicaria na busca por mais consistência, tornando mais custosa a mudança (Porter, 1989), o que já ocorre se consideramos que a firma é parte de um contexto que possui outros participantes: o sistema de atividades (Shank \& Govindarajan, 1992).

Diante do exposto, deduz-se que qualquer estrutura a ser adotada demandará custos ex ante, portanto $\mathrm{C}_{\mathrm{MI}}$ apresenta-se como um custo de transação e, de acordo com $\mathrm{H} 1$, afeta o processo de criação de valor na cadeia. Essas estruturas, por definição, são constituídas a partir da constatação da natureza dos ativos envolvidos e a isto acrescenta-se que as estruturas só serão capazes de economizar custos à medida que forem organizadas de forma discriminada para cada transação, constituindo-se como simples ou complexas de acordo com as dimensões envolvidas (Williamson, 1985). CMI será > 0 , pois a existência de ativos suscita o oportunismo e sujeita à racionalidade limitada na constituição de salvaguardas, sendo, $\mathrm{CA}_{i}, \mathrm{COP}_{\mathrm{i}, \mathrm{i}+1}, \mathrm{CRL_{i } , \mathrm { i } + 1}$ e $\mathrm{CEA}_{\mathrm{i}, \mathrm{i}+1}>0$. Se a forma organizacional deve buscar a melhor adaptação, deve ser flexível para se adaptar à incerteza e ao mesmo tempo segura para proteger os ativos envolvidos. Logo, para otimizar o valor criado pela cadeia, é necessária a adoção de mais de uma matriz institucional que tornará o custo do elo mais econômico. Assim, tem-se a Hipótese (H2a):

H2a: o custo do elo é positivamente relacionado com o custo da matriz institucional. 
Numa estrutura de custos, formas organizacionais são consideradas como custos fixos, uma vez que não se alteram em razão da quantidade produzida. O impacto produzido na curva de custos totais pelas formas pode ser minimizado pela adoção da economia de escopo (EE) em que o custo total (CT) do desempenho interno das atividades seja menor que o custo do desempenho feito pela estrutura de mercado (Besanko, Dranove, Shanley, \& Schaeffer, 2004).

Em atividades que já desenvolvem vantagem de custo, o potencial para redução de custo está relacionado à competência essencial da firma (Prahalad \& Hamel, 1990), indicando que quanto mais estreito for o escopo, mais econômica será a cadeia, o que leva a crer que a realização de novas atividades que possam ser desenvolvidas a partir das economias de escopo dentro de uma hierarquia tendem a ser mais econômicas do que outras, mesmo considerando a escala de produção. Da mesma forma, segundo Besanko, Dranove, Shanley e Schaeffer (2004), a realização de atividades fora do escopo encontra barreira no custo médio, que é reduzido com a introdução de economias de escala (ES), nas quais o custo médio do desempenho da atividade é menor que seu custo marginal.

Economias de escopo e escala influenciam o $\mathrm{C}_{\mathrm{MI}}$, conforme equação 4, sugerindo que atividades especificamente estratégicas devem ser desempenhadas numa hierarquia e atividades genéricas devem ser desempenhadas na forma híbrida, derivando a Hipótese (H2b):

$$
\Delta \mathrm{C}_{\mathrm{MI}=} \mathrm{C}_{\mathrm{MI}} \cdot(\mathrm{EE}+\mathrm{ES})
$$

H2b: o custo da matriz institucional é positivamente relacionado com as economias de escala e escopo da firma.

Se a vantagem competitiva pode ser verificada através do desempenho das atividades (Porter, 1985), na ECT, ela é obtida pela melhor forma organizacional, melhor incentivo interno, controle e alinhamento entre as interfaces contratuais (Williamson, 1991a) proporcionados pela estrutura de governança (Williamson, 1996). As estruturas de governança e sua continuidade são responsáveis pelos desempenhos diferenciados entre as firmas e sua eficiência é determinada pelos esforços locais para mitigação de conflitos e obtenção de ganhos mútuos no ambiente institucional (Williamson, 1999, 2005).

Por um lado, a estrutura organizacional mais adequada para gerenciamento de uma cadeia de valor é a estrutura funcional (U), dada a divisão da firma em funções classificadas mediante sua agregação de valor ao produto final que ocorre pela crença de que as atividades da cadeia são interdependentes entre si, porém não independentes (Porter, 1989). Por outro lado, a utilização de uma matriz institucional híbrida possibilita o aumento do escopo de segmento, significando que a firma vai operar com maior variedade de bens ou serviços (Porter, 1989), conduzindo-a à diversificação e ao aumento nos custos de transação, uma vez que mais transações serão desempenhadas. Neste caso, surge a possibilidade da utilização de uma estrutura multidivisional $(\mathrm{M})$, conforme sugestão de Williamson (1991a), ou independente (B), como ocorre com os business groups (Colpan \& Hikino, 2010). Considerando que para cada negócio deverá ser definido um posicionamento, o aumento do escopo e a utilização da matriz híbrida farão com que o custo fixo das estruturas organizacionais $\left(\mathrm{C}_{\mathrm{EO}}\right)$ varie de acordo com o número de negócios desempenhados pela firma.

O posicionamento também possui como consequência o desenvolvimento de estrutura com requisitos de controle e coordenação (Porter, 1985), porém numa firma multinegócios os níveis de agrupamentos são maiores porque além de divididos por grupos funcionais estes grupos ainda são específicos por negócio, aumentando a possibilidade de problemas de agência, complexidade da estrutura e a dificuldade em coordenar estes grupos para atingir os objetivos da corporação (Sah \& Stiglitz, 1986), fazendo com que a perda de controle limite o crescimento da firma (Natividad, 2011), aumentando o custo da estrutura funcional $\left(\mathrm{UC}_{\mathrm{EO}}\right.$ ). Conforme a Equação 2, o valor total da cadeia considera valores e custos individuais das atividades e custos de transação o que só será possível de ser mensurado no caso de uma diversificação, se as atividades forem tratadas como centro de custos independentes nos moldes de uma estrutura multidivisional. 
Contingências internas não são reconhecidas na economia dos custos de transação (Hill, 1985) e, neste sentido, aponta-se que a utilização da estrutura multidivisional tem sido criticada por estudos como o de Mahoney (1992), que inclui perspectivas de eficiência, poder e dependência. Contribuições com base em testes empíricos indicaram que a estrutura multidivisional é uma estrutura adequada a um contexto de diversificação moderada e está sujeita a contingências internas (Hoskisson, Hill, \& Kim, 1993). Porém estudos recentes apontam a eficiência das estruturas multidivisionais, pois favorecem o controle organizacional e, assim, continuam sendo consideradas respostas gerenciais aos custos de transação (Bucheli, Mahoney, \& Vaaler, 2010). Considerando que, em ambientes de rápidas mudanças, a escolha do modo de governança poderia seguir uma abordagem contingencial (Lin, Yeh, $\& \mathrm{Li}, 2011)$, a $M$-form é a forma organizacional mais adequada quando há possibilidade de negociação entre principal e seus agentes (Bao \& Wang, 2011), tratando-se cada atividade especificamente estratégica como um centro de custo separado; no entanto, em se tratando de alta diversificação, onde ocorre uma natural diminuição da interdependência entre as atividades e estudos como o de Aggarwal, Siggelkow e Singh (2011) indicam a utilização de uma gestão independente, a exemplo do que ocorre nos business group.

Considerando a ECT, tais achados justificam-se pela necessidade de informações que representam custo $\left(\mathrm{C}_{\mathrm{INF}}\right)$, reforçando o uso da estrutura independente $\left(\mathrm{B}_{\mathrm{CEO}}\right)$, que é mais econômica por não demandar de tais informações, conforme sugere a equação 5.

$U C_{E O}=C_{M I}+C_{I N F}$

$B C_{E O}=C_{M I}$

Se $C_{I N F}=0$, então $U C_{E O}=B C_{E O}$ (pode-se usar a estrutura funcional ou independente)

Se $C_{I N F}>0$, então $U C_{E O}>B C_{E O}$ (o uso da estrutura independente é mais eficiente)

A gestão independente de cada atividade também favorece sistemáticas de controle, atuando positivamente sobre racionalidade limitada e oportunismo que são majorados na medida em que aumentam especificidade dos ativos e informações são perdidas porque o processo de tramitação é maior, de forma que a gestão independente da hierarquia maximizará o valor criado. Assim, apresentase a Hipótese (H3):

H3: o custo do elo é positivamente relacionado com o custo da estrutura organizacional.

A Figura 4 ilustra, em destaque, a esquematização das relações causais entre constructos descrita nas hipóteses e que compõe o aparato proposto neste ensaio. A partir da adoção do strategizing e economizing como condições necessárias para criação de valor, que é o objetivo da firma, tem-se o Valor Total Criado como variável dependente no arranjo conceitual. A visão porteriana traz como variáveis independentes o Valor das Atividades e o Custo das Atividades que, por não serem objeto de estudo deste ensaio, foram sucintamente representadas. As hipóteses desenvolvidas apresentam o Custo do Elo como a variável independente acrescida ao arranjo porteriano, tendo Estrutura Organizacional e Matriz Institucional como variáveis moderadoras, assumindo ainda que Economias de Escala e Escopo guardam relação negativa com a Matriz Institucional. A operacionalização desta proposta é sugerida e sumarizada na Tabela 1, que contém a descrição dos constructos e respectivas variáveis observadas ou proxies sugeridas. 


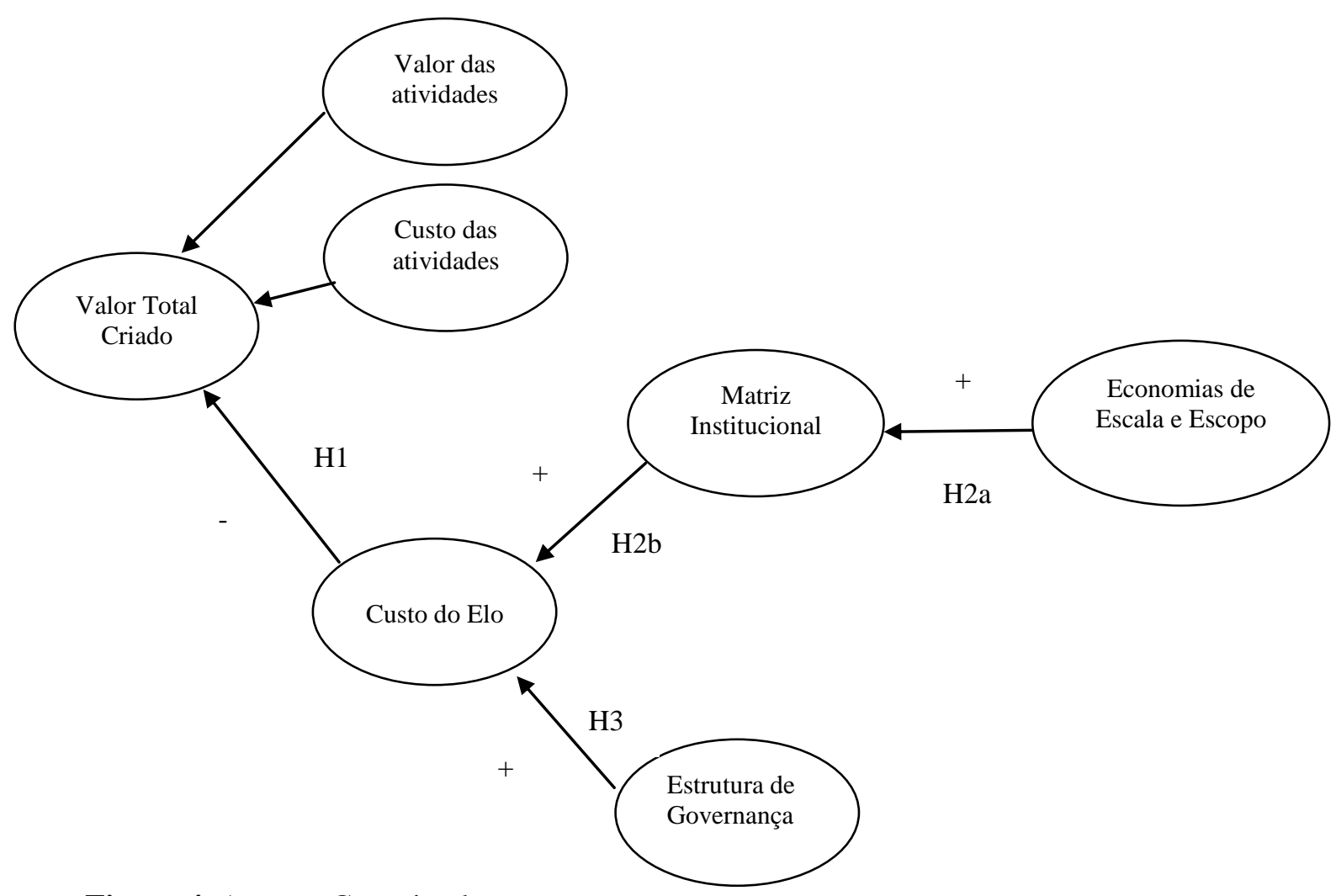

Figura 4. Aparato Conceitual.

Fonte: Elaborado pelos autores.

Tabela 1

Descrição dos Construtos

\begin{tabular}{|c|c|c|c|}
\hline Constructos & Tipo de variável & Definição & Variável observada \\
\hline Valor & Dependente & $\begin{array}{l}\text { É o lucro da firma, obtido através da diferença entre } \\
\text { o valor e o custo criados na cadeia (Porter, 1989). }\end{array}$ & Lucro \\
\hline Custo do elo & Independente & $\begin{array}{l}\text { Custo inerente à transferência de um bem entre } \\
\text { atividades (Coase, 1937). }\end{array}$ & $\begin{array}{l}\text { Custo de inputs } \\
\text { intermediários/ custo } \\
\text { do enforcement }\end{array}$ \\
\hline $\begin{array}{l}\text { Matriz } \\
\text { Institucional }\end{array}$ & Moderadora & $\begin{array}{l}\text { Forma com a qual as atividades serão } \\
\text { desempenhadas pela firma, usando ou não a estrutura } \\
\text { de mercado (Williamson, 1981, 1991a, 1991b). }\end{array}$ & Capital de giro \\
\hline $\begin{array}{l}\text { Economias de } \\
\text { Escala e Escopo }\end{array}$ & Moderadora & $\begin{array}{l}\text { Economias de escala são aquelas que ocorrem } \\
\text { quando o custo médio do desempenho da atividade é } \\
\text { menor que seu custo marginal. Economias de escopo } \\
\text { são aquelas que ocorrem quando o custo total do } \\
\text { desempenho interno das atividades seja menor que o } \\
\text { custo do desempenho feito pela estrutura de mercado } \\
\text { (Besanko et al., 2004). }\end{array}$ & $\begin{array}{l}\text { Custos } \\
\text { operacionais/ } \\
\text { capital de giro }\end{array}$ \\
\hline $\begin{array}{l}\text { Estrutura de } \\
\text { governança }\end{array}$ & Moderadora & $\begin{array}{l}\text { É a forma organizacional usada para coordenar as } \\
\text { atividades dentro da firma (Williamson, 1991a) }\end{array}$ & $\begin{array}{l}\mathrm{B}, \mathrm{U} \text {-form/variável } \\
\text { dummy }\end{array}$ \\
\hline
\end{tabular}

Nota. Fonte: elaborado pelos autores. 


\section{Implicações da Proposta}

O framework porteriano aponta que somente o desempenho das atividades é que determina a criação de valor (Porter, 1991), mas as atividades precisam exibir consistência interna, ou seja, serem coordenadas num nexo de transações. Não só a consistência interna afeta o valor criado, conforme afirmam Porter e Siggelkow (2008), mas também o desempenho das transações entre elas; se os elos são transações e, como tal, possuem custos, então, a criação de valor também reside no custo dos elos, consistindo na primeira implicação da proposta.

A segunda implicação baseia-se no questionamento sobre a efetividade de uma atividade ser afetada pelo desempenho de outra (Porter, 1991). O aparato proposto evidencia que pode haver dissipação de valor no elo, o que compromete as atividades das quais ele seja o ponto de contato, fazendo com que o desempenho das atividades também seja afetado pelos custos do elo e não só pelo desempenho da atividade antecessora.

A terceira implicação refere-se ao entendimento de que as atividades são a unidade fundamental do comportamento de custo da firma e da vantagem competitiva, pois, partindo do princípio que transações estão presentes inclusive na execução de uma atividade (Coase, 1937), ao incluir-se esta perspectiva no aparato proposto, neste ensaio, refuta-se a hipótese de que a escolha de um conjunto único de atividades garanta a criação de valor (Porter, 1991, 1996).

Tais implicações baseiam-se na ideia de que a eficiência de uma transação é dada pelo seu menor custo propiciado pela estrutura de governança mais adequada (Williamson, 1991b), incorporada no arranjo proposto. Ao utilizarmos o exemplo das cadeias de valor globais, torna-se mais evidente que, ao longo de toda cadeia, certo nível de governança é necessário (Morrison, Pietrobelli, \& Rabellotti, 2008) devido às dimensões envolvidas que são consistentes com as definições da ECT, dessa maneira, contrapondo-se aos argumentos de Ito, Hayashi, Gimenez e Fensterseifer (2012), os quais colocam a transação como troca econômica (bem por preço); e governança como um pacto de valor, e não como uma estrutura de salvaguarda, fugindo da lógica da ECT.

Quanto às implicações gerenciais, deve-se atentar porque a dissipação de valor nos elos acentuase à medida que se tratam de elos externos, ou seja, elos que envolvem estruturas de mercado, pois governam transações maiores em termos de volume financeiro, número de produtos ou componentes envolvidos (Batenburg, Raub, \& Snijiders, 2003); e o oportunismo está ligado à complexidade do produto, suas características não observáveis diretamente (Buskens, Batenburg, \& Wessie, 2003) e ao tamanho da transação desempenhada. A eficiência dos mecanismos preventivos contra a dissipação de valor utilizado nos elos pode representar uma redução dos riscos envolvidos (Buskens, Raub, \& Snijders, 2003), mas deve-se considerar que a partilha do valor é inevitável entre os participantes da indústria (Porter \& Kramer, 2011).

Mecanismos preventivos constituem-se na ECT como instrumentos formais - os contratos -, porém estão sujeitos à racionalidade limitada (Williamson, 1985), sendo impossível concentrar tudo o que é relevante no ex ante. Também, ocorre que tais mecanismos, constituídos para prevenir ou minimizar a dissipação de valor, presumem constância nos contextos presente e futuro, o que de fato não ocorre (Batenburg et al., 2003) e, por isso, contratos precisam ter flexibilidade para serem revisados. Assim, em relações de longo prazo, mecanismos informais podem se apresentar como formas de se obter maior eficiência dos elos.

\section{Conclusão}

Este ensaio teve como objetivo incorporar elementos da ECT na visão porteriana, assim, propondo um aparato conceitual para criação de valor. Esta integração encontra barreiras, pois, além 
de possuírem unidades de análise diferentes, as duas perspectivas se posicionam de formas diferentes em relação à orientação para lucratividade, estabelecendo uma discussão entre strategizing e economizing. Identificou-se que a cadeia de valor/sistema de atividades é composta por duas unidades básicas de ativos coordenados, as atividades e os elos. O levantamento bibliográfico apresentou estudos sobre as atividades e sua contribuição para criação de valor de acordo com a contextualidade (Porter \& Siggelkow, 2008), mas que continuam ignorando o papel dos elos no processo de criação de valor.

A partir do uso da perspectiva dos custos de transação, foi possível concluir que a criação de valor também é afetada pelo elo, sendo determinada pela relação entre valor criado pela atividade e seus custos mais o custo do elo. A ECT ainda contribui com a análise de criação de valor na cadeia na medida em que insere, no framework, as crenças de comportamentos e os atributos das transações como determinantes do custo. Em contrapartida, a utilização das ideias da cadeia de valor/sistema de atividades permite preencher uma lacuna que é deixada ao se utilizar somente a ECT para a análise: não só transações possuem custo, mas as atividades que estas transações governam.

A tentativa de integrar a visão porteriana com a ECT resultou num aparato teórico que apresenta três dimensões para a criação de valor: o valor criado pelas atividades, o custo das atividades e o custo dos elos. Explorando a dimensão do custo dos elos, a presente proposta enfatiza o papel das matrizes institucionais e estruturas de governanças para obterem interfaces tecnológicas mais econômicas, maximizando o valor criado na cadeia. Estas contribuições são relevantes porque viabilizam mais completude ao processo de análise da firma, refinando-o por meio da identificação de elementos anteriormente não incorporados na abordagem de Porter nem na ECT.

Como recomendação de trabalhos futuros, sugere-se o teste empírico das relações hipotetizadas, estabelecendo, a partir de decisões metodológicas, um design apropriado para coleta e análise de dados. Reconhece-se as dificuldades na coleta de dados em relação à estrutura de governança que, a princípio, é apresentada como uma variável nominal tratada como dummy e cujo acesso provavelmente seja feito através de levantamento documental junto às firmas componentes de uma amostra. Num esforço para integrar a economia dos custos de transação com a economia industrial, este ensaio procurou contribuir com o debate sobre um dos frameworks mais conhecidos no campo da estratégia, mas ainda pouco explorado teórica e empiricamente nos estudos acadêmicos.

Como recomendação para trabalhos futuros, sugere-se o teste empírico das relações hipotetizadas, estabelecendo um design apropriado para coleta e análise de dados. Inicialmente são sugeridas duas opções metodológicas: (a) seguir as recomendações de Porter e Siggelkow (2008) que sugerem estudos longitudinais e multinível (incorporando efeitos indústria e firma) para identificar as implicações de performance do sistema de valor incorporando, de acordo com a proposta deste artigo, os custos dos elos; (b) a exemplo das contribuições de Dutta e John (1995), Sutcliff e Zaheer (1998) e Macher e Richman (2008), desenhar um experimento aleatório no qual, em um ambiente controlado, o pesquisador possa manipular as variáveis independentes - valor das atividades, custo das atividades, custo do elo - e assim testar a influências destas sobre a variável dependente - valor total criado mediante a observação de grupos de controle e teste e suas respectivas opções dentro do sistema de atividades. Num esforço para integrar a economia dos custos de transação com a economia industrial, este ensaio procurou contribuir com o debate sobre um dos frameworks mais conhecidos no campo da estratégia, mas ainda pouco explorado teórica e empiricamente nos estudos acadêmicos.

\section{Referências}

Aggarwal, V. A., Siggelkow, N., \& Singh, H. (2011). Governing collaborative activity: interdependence and the impact of coordination and exploration. Strategic Management Journal, 32(7), 705-730. doi: 10.1002/smj.900 
Alchian, A. A., \& Demsetz, H. (1972). Production, information costs, and economic organization. The American Economic Review, 62(5), 777-795. doi: 10.2307/1815199

Bao, T., \& Wang, Y. (2012). Incomplete contract, bargaining and optimal divisional structure. Journal of Economics, 107(1), 81-96. doi: 10.1007/s00712-011-0258-0

Batenburg, R. S., Raub, W., \& Snjiders, C. C. P. (2003). Contacts and contracts: dyadic embeddedness and the contractual behavior of firms. Research in the Sociology of Organizations, 20, 135-188. doi: 10.1016/S0733-558X(02)20006-3

Besanko, D., Dranove, D., Shanley, M., \& Shaefer, S. (2004). The economics of strategy. New Jersey: John Wiley and Sons.

Bucheli, M., Mahoney, J. T., \& Vaaler, P. M. (2010). Chandler's living history: the visible hand of vertical integration in nineteenth century America viewed under a twenty-first century transaction costs economics lens. Journal of Management Studies, 47(5), 859-883. doi: 10.1111/j.1467-6486.2010.00927.x

Buskens, V., Batenburg, R. S., \& Wessie, J. (2003). Embedded partner selection in relations between firms. Research in the Sociology of Organization, 20, 107-133. doi: 10.1016/S0733$558 \mathrm{X}(02) 20005-1$

Buskens, V., Raub, W., \& Snjiders, C. (2003). Theoretical and empirical perspectives on the governance of relations in markets and organizations. Research in the Sociology of Organization, 20, 1-18. doi: 10.1016/S0733-558X(02)20001-4

Chatain, O., \& Zemsky, P. (2011). Value Creation and Value Capture with Frictions. Strategic Management Journal, 32(11), 1206-1231.

Coase, R. (1937). The nature of the firm. Economica, 4(16), 386-405. doi: 10.1111/j.14680335.1937.tb00002.x

Colpan, A. M., \& Hikino, T. (2010). Foundations of business groups: towards an integrated framework. In A. M. Colpan, T. Hikino, \& J. R. Lincoln (Eds.), The oxford handbook of business groups (pp. 15-66). New York: Oxford University Press.

Dutta, S., \& John, G. (1995). Combining lab experiments and industry data in transaction cost analysis: the case of competition as a safeguard. Journal of Law, Economics and Organization, 11(1), 87-111.

Gereffi, G., Humphrey, J., \& Sturgeon, T. (2005). The governance of global value chains. Review of International Political Economy, 12(1), 78-104. doi: 10.1080/09692290500049805

Hill, C. W. L. (1985). Oliver Williamson and the m-form firm: a critical review. Journal of Economic Issues, 19(3), 731-751. Recuperado de http://www.jstor.org/stable/4225617

Hoskisson, R. E., Hill, C. W. L., \& Kim, H. (1993). The multidivisional structure: organizational fossil or source of value? Journal of Management, 19(2), 269-298. doi: 10.1016/01492063(93)90055-R

Ito, N. C., Hayashi, P., Gimenez, F. A. O., \& Fensterseifer, J. E. (2012). Valor e vantagem competitiva: buscando definições, relações e repercussões. Revista de Administração Contemporânea, 16(2), 290-307. Recuperado de http://www.scielo.br/pdf/rac/v16n2/v16n2a08.pdf. doi: 10.1590/S1415-65552012000200008

Klein, B., Crawford, R. G., \& Alchian, A. (1978). Vertical Integration appropriable rents, and the competitive contracting process. Journal of Law and Economics, 21(2), 297-326. doi: $10.1086 / 466922$ 
Lin, Y., Yeh, K. S., \& Li, S. (2011). Change of governance in the organization value chain: the case of the high-tech industries in Taiwan. Corporate Governance: an International Review, 19(2), 169-182. doi: 10.1111/j.1467-8683.2011.00847.x

Lindenberg, S. (2003). The cognitive side of governance. In V. Buskens, W. Raub, \& C. Snijders (Eds.), The governance of relations in markets and organizations (pp. 47-76). Bingley: Emerald Group Publishing Limited.

Macher, J. T., \& Richaman, B. D. (2008). Transaction cost economics: an assessment of empirical research in the social sciences. Business and Politics, 10(1), 1-63. doi: 10.2202/14693569.1210 .

Mahoney, J. T. (1992). The choice of organizational form: vertical financial ownership versus other methods of vertical integration. Strategic Management Journal, 13(8), 559-584. doi: $10.1002 / \mathrm{smj} .4250130802$

Morrison, A., Pietrobeli, C., \& Rabellotti, R. (2008). Global value chains and technological capabilities: a framework to study learning and innovation in developing countries. Oxford Development Studies, 36(1), 39-58. doi: 10.1080/13600810701848144

Natividad, G. (2011). The organizational efficiency of internal capital markets [AFA 2009 San Francisco Meetings Paper]. Social Science Research Network. Recuperado de http://ssrn.com/paper=1107555

Patnaik, R., \& Sahoo, P. K. (2009). Understanding value chain for growth: a case of Indian wine industry. Journal of Supply Chain Management, 6(3/4), 27-40.

Porter, M. E. (1985). Estratégia competitiva. Rio de Janeiro: Campus.

Porter, M. E. (1989). Vantagem competitiva. Rio de Janeiro: Campus.

Porter, M. E. (1990). The competitive advantage of nations. Harvard Business Review, 68(2).73-93.

Porter, M. E. (1991). Towards a dynamic theory of strategy. Strategic Management Journal, 12(s2), 95-117. doi: 10.1002/smj.4250121008

Porter, M. E. (1996). What is strategy? Harvard Business Review, 74(6), 61-78.

Porter, M. E. (1999). Competição: estratégias competitivas essenciais (6a ed.). Rio de Janeiro: Editora Campus.

Porter, M. E., \& Kramer, M. R. (2011). Creating shared value. Harvard Business Review, 89(1/2), 6277.

Porter, M. E., \& Siggelkow, N. (2008). Contextuality within activity systems and sustainability of competitive advantage. Academy of Management Perspectives, 22(2), 34-56. doi: 10.5465/AMP.2008.32739758

Powell, T. C. (2001). Competitive advantage: logical and philosophical considerations. Strategic Management Journal, 22(9), 875-888. doi: 10.1002/smj.173

Prahalad, C., \& Hamel, G. (1990). The core competence of the corporation. Harvard Business Review, 68(3), 79-91.

Roodhoof, F., \& Warlop, L. (1999). On the role of sunk costs and asset specificity in outsourcing decisions: a research note. Accounting, Organizations and Society, 24(4), 365-369. doi: 10.1016/S0361-3682(98)00069-5 
Sah, R. K., \& Stiglitz, J. E. (1986). The architecture of economic system: hierarchies and polyarchies. The American Economic Review, 76(4), 716-727. doi: 10.2307/1806069

Shank, J. K., \& Govindarajan, V. (1992). Strategic cost management: the value chain perspective. Journal of Management Accounting Research, 4(4) 179-197.

Shapiro, J., Singhal, V., \& Wagner, S. (1993). Optimizing the value chain. Interfaces, 23(2), 102-117. Recuperado de http://www.jstor.org/stable/25061732

Stabell, C. B., \& Fjestad, O. D. (1998). Configuring value for competitive advantage: on chains, shops and networds. Strategic Management Journal, 19(5). 413-437. doi: 10.1002/(SICI)10970266(199805)19:5<413::AID-SMJ946>3.0.CO;2-C

Sutcliffe, K. M., \& Zaheer, A. (1998). Uncertainly in the transaction environment: an empirical test. Strategic Management Journal, 19(1), 1-23. doi: 10.1002/(SICI)10970266(199801)19:1<1::AID-SMJ938>3.0.CO;2-5

Voss, T. (2003). The rational choice approach to an analysis of intra and inteorganizational governance. Research in the Sociology of Organization, 20,21-46. doi: 10.1016/S0733$558 \mathrm{X}(02) 20002-6$

Williamson, O. E. (1981). The economics of organization: the transaction cost approach. The American Journal of Sociology, 87(3), 548-577. doi: 10.2307/2778934

Williamson, O. E. (1985). The economic institutions of capitalism. New York: Free Press.

Williamson, O. E. (1991a). Comparative economic organization: the analysis of discrete structural alternatives. Administrative Science Quarterly, 36(2), 269-296. doi: 10.2307/2393356

Williamson, O. E. (1991b). Strategizing, economizing, and economic organization. Strategic Management Journal, 12(S2), 75-94. doi: 10.1002/smj.4250121007

Williamson, O. E. (1996). The mechanisms of governance. New York: Oxford.

Williamson, O. E. (1999). Governance and competence perspectives. Strategic Management Journal, 20(12), 1087-1108. doi: 10.1002/(SICI)1097-0266(199912)20:12<1087::AIDSMJ71>3.3.CO;2-Q

Williamson, O. E. (2005). The economics of governance. American Economic Association, 95(2), 118.

Williamson, O. E. (2008). Outsourcing: transaction cost economics and supply chain management. Journal of Supply Chain Management, 44(2), 6-16. doi: 10.1111/j.1745-493X.2008.00051.x 